\title{
DOA Estimation of Cylindrical Conformal Array Based on Geometric Algebra
}

\author{
Minjie Wu, Xiaofa Zhang, Jingjian Huang, and Naichang Yuan \\ Department of Electronic Science and Engineering, National University of Defense Technology, Deya Road 109, \\ Changsha 410073, China
}

Correspondence should be addressed to Minjie Wu; wmj601@nuaa.edu.cn

Received 7 July 2016; Revised 18 October 2016; Accepted 2 November 2016

Academic Editor: Youssef Nasser

Copyright (c) 2016 Minjie Wu et al. This is an open access article distributed under the Creative Commons Attribution License, which permits unrestricted use, distribution, and reproduction in any medium, provided the original work is properly cited.

Due to the variable curvature of the conformal carrier, the pattern of each element has a different direction. The traditional method of analyzing the conformal array is to use the Euler rotation angle and its matrix representation. However, it is computationally demanding especially for irregular array structures. In this paper, we present a novel algorithm by combining the geometric algebra with Multiple Signal Classification (MUSIC), termed as GA-MUSIC, to solve the direction of arrival (DOA) for cylindrical conformal array. And on this basis, we derive the pattern and array manifold. Compared with the existing algorithms, our proposed one avoids the cumbersome matrix transformations and largely decreases the computational complexity. The simulation results verify the effectiveness of the proposed method.

\section{Introduction}

A conformal antenna is an antenna that conforms to a prescribed shape. The shape can be some part of an airplane, high-speed missile, or other vehicle [1]. Their benefits include reduction of aerodynamic drag, wide angle coverage, and space-saving [2]. Nevertheless, due to the complex curved surface structure, the pattern of each antenna is inconsistent. Thus, the conformal array can no longer be regarded as a simple isotropic one. The pattern multiplication theorem is not available as well. Most classical DOA estimation algorithms cannot be directly transplanted to such scene.

In recent years, there has been a considerable interest in estimating DOAs for conformal array. Milligan used Euler rotation angles to find the patterns with elements in a conformal array that requires one to rotate not only the direction but also the polarization [3]. In [2], Wang et al. proposed a uniform method for the element polarized pattern transformation of arbitrary 3D conformal arrays based on Euler rotation. Yang et al. introduced a conformal array DOA algorithm with an unknown source number; the method was realized by virtue of the pseudo expected signal [4]. However, the root mean square error (RMSE) deteriorated severely when the number of snapshots was small. Up to the present, we have observed that most of DOA estimation algorithms for conformal array are based on the Euler rotation transformation which converts the local coordinate system to the global coordinate system. Though the Euler rotation angle is a useful tool for spatial rotation transformation [5], a huge amount of computation is incurred.

Geometric algebra is the largest possible associative algebra that integrates all algebraic systems (algebra of complex numbers, matrix algebra, quaternion algebra, etc.) into a coherent mathematical language [6]. Three-dimensional pattern analysis of arbitrary conformal arrays using the mathematical framework of the geometric algebra was introduced [7]. Nevertheless, this mathematical language was not transplanted to the DOA estimation. In [8], Zou et al. took several elements as a new one and transformed the original array into another regular array to estimate the DOA. However, this method was only suitable for some particular array structures. Combining the MUSIC with geometric algebra to solve the DOA estimation has not been addressed in the literature. In this paper, we fill this gap and study the problem based on the cylindrical conformal array. Compared with the existing methods, the proposed one has three main 
TABLE 1: Properties of the outer product.

Property
Antisymmetry
Scaling
Distributivity
Associativity

(a)

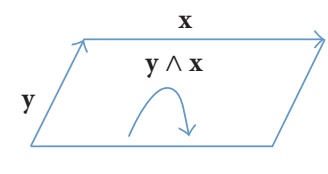

(b)
FIGURE 1: The geometry of outer product (a) $\mathbf{x} \wedge \mathbf{y}$ and (b) $\mathbf{y} \wedge \mathbf{x}$.

advantages. Firstly, it does not need to calculate the rotation matrices and therefore has a much lower computational complexity. Subsequently, it is not limited to the cylindrical conformal array due to its strong commonality. Finally, it can still work effectively even when the number of polarized signals is larger than that of the array elements.

The structure of this paper is as follows. In Section 2, the rotors in geometric algebra which establishes the mathematical knowledge of transformation is briefly introduced. In Section 3, we derive the cylindrical conformal array manifold using rotors and present the GA-MUSIC algorithm. In addition, to better explain the superiority of GA-MUSIC in reducing the computational complexity, we briefly introduce the Euler angle and compare it with the proposed algorithm. Simulations using the proposed method for cylindrical conformal array are given in Section 4. Finally, the conclusions are drawn.

\section{Rotors in Geometric Algebra}

Geometric algebra was first introduced by the British mathematician, named Clifford, in the nineteenth century. He constructed the geometric product by combining the inner product with the outer product. The main advantage of the geometric algebra is embodied in processing the rotation transformation [9]. Various rotations can be described by an element called the rotor. A rotor is more general than an Euler rotation angle because a rotor can be used in an arbitrary dimensional space.

We begin by introducing a new product between vectors that we call the outer product. Let us use the wedge symbol " $\wedge$ " to denote outer product with the properties listed in Table 1.

The outer product is regarded as the "addition operator" of subspaces, in that the outer product $\mathbf{x} \wedge \mathbf{y}$ spans the subspace that $\mathbf{x}$ and $\mathbf{y}$ span together, as long as $\mathbf{x}$ and $\mathbf{y}$ are independent. The geometry is illustrated in Figure 1.

Next, we will introduce the fundamental product of the geometric algebra, namely, geometric product. It is simply the sum of the outer and inner product:

$$
\mathbf{x y}=\mathbf{x} \bullet \mathbf{y}+\mathbf{x} \wedge \mathbf{y} .
$$

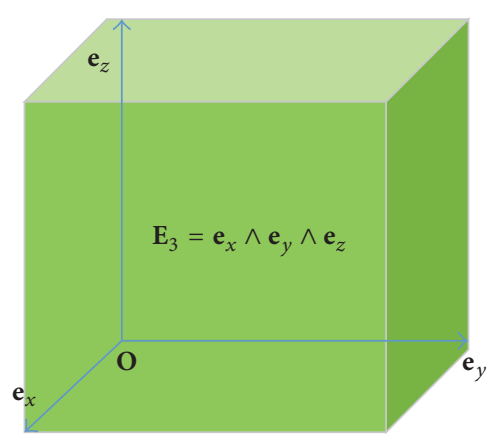

FIGURE 2: The geometry of 3-blade.

Reversing the order of $\mathbf{x}$ and $\mathbf{y}$ in (1), by means of the symmetry of the inner product and the antisymmetry of the outer product, it follows that

$$
\mathbf{y x}=\mathbf{x} \bullet \mathbf{y}-\mathbf{x} \wedge \mathbf{y}
$$

Thus, combining (1) with (2), the inner product and the outer product can be uniformly represented by the geometric product:

$$
\begin{aligned}
& x \bullet y=\frac{x y+y x}{2} \\
& x \wedge y=\frac{x y-y x}{2}
\end{aligned}
$$

Generally, we call an outer product of $k$ vectors a $k$-blade. The value of $k$ is called the grade of the blade. Specifically, the top-grade blades $\mathbf{E}_{n}$ in an $n$-dimensional space are called pseudoscalars. In principle, blades are just elements of the geometric algebra. To be useful for doing geometry, blades can be interpreted as subspaces. $\mathbf{x} \wedge \mathbf{y}$ is a 2 -blade as shown in Figure 1(a). By introducing vector $\mathbf{z}$, we can construct the 3blade $\mathbf{x} \wedge \mathbf{y} \wedge \mathbf{z}$, which coincides with the Cartesian coordinate system. As shown in Figure 2, the unit vectors $\mathbf{e}_{x}, \mathbf{e}_{y}$, and $\mathbf{e}_{z}$, in that order, form a right-handed Cartesian coordinate system. $\mathbf{E}_{3}$ is the pseudoscalar, relative to the origin denoted by $\mathbf{O}$. The 3 -blade is drawn as a parallelepiped. The volume depicts the weight of the 3-blade, but, in principle, blades have no specific shape.

As shown in Figure 3, vector $\mathbf{y}$ is obtained by rotating vector $\mathbf{x}$ with $\theta$. We can regard the rotation as two consecutive reflections, first in $\mathbf{a}$ and then in $\mathbf{b}$. The expression that reflects $\mathbf{x}$ in the line with direction $\mathbf{a}$ is

$$
\mathbf{x}^{\prime}=\mathbf{a x a}=2(\mathbf{a} \cdot \mathbf{x}) \mathbf{a}-\mathbf{x} .
$$

In (4), a must be unit length. We give the detailed derivation process in Appendix. The regularity condition can be dropped by using an inverse geometric product, as in

$$
\mathbf{x}^{\prime}=\mathbf{a x a} \mathbf{a}^{-1}=2(\mathbf{a} \bullet \mathbf{x}) \mathbf{a}^{-1}-\mathbf{x},
$$

where

$$
\mathbf{a}^{-1}=\frac{\mathbf{a}}{\mathbf{a} \cdot \mathbf{a}}
$$

with $(\cdot)^{-1}$ representing the inverse operator. 


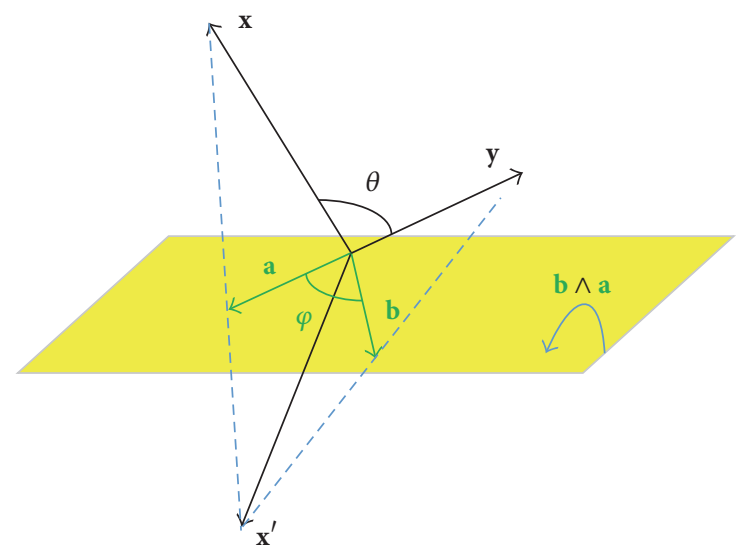

FIGURE 3: Rotation of vector $\mathbf{x}$.

Then, $\mathbf{y}$ can be obtained by reflecting $\mathbf{x}^{\prime}$ in the line with direction $\mathbf{b}$ :

$$
\mathbf{y}=\mathbf{b x} \mathbf{x}^{\prime} \mathbf{b}^{-1}=\mathbf{b a x a}^{-1} \mathbf{b}^{-1}=(\mathbf{b a}) \mathbf{x}(\mathbf{b a})^{-1}=\mathbf{R x R ^ { - 1 }} .
$$

Thus, we can identify $\mathbf{R}$ as the rotor. To proceed further, we rewrite $\mathbf{R}$ according to the definition of the geometric product:

$$
\mathbf{R}=\mathbf{b} \mathbf{a}=\mathbf{b} \cdot \mathbf{a}+\mathbf{b} \wedge \mathbf{a} .
$$

Here, we consider the case that the vectors are unit length. This assumption is reasonable, because the basic vectors of the Cartesian coordinate system satisfy it as well. The geometric product of $\mathbf{b} \wedge \mathbf{a}$ itself is

$$
\begin{aligned}
(\mathbf{b} \wedge \mathbf{a})(\mathbf{b} \wedge \mathbf{a}) & =(\mathbf{b} \mathbf{a}-\mathbf{b} \bullet \mathbf{a})(\mathbf{b} \bullet \mathbf{a}-\mathbf{a b}) \\
& =\mathbf{b} \bullet \mathbf{a}(\mathbf{b} \mathbf{a}+\mathbf{a b})-(\mathbf{b} \bullet \mathbf{a})^{2}-\mathbf{b a a b} \\
& =\mathbf{b} \cdot \mathbf{a}(\mathbf{2} \mathbf{b} \bullet \mathbf{a})-(\mathbf{b} \cdot \mathbf{a})^{2}-\mathbf{b}(\mathbf{a a}) \mathbf{b} \\
& =(\mathbf{b} \cdot \mathbf{a})^{2}-\mathbf{b} \mathbf{b}=\cos ^{2} \theta-1=-\sin ^{2} \theta
\end{aligned}
$$

Thus, we define the 2-blade $\mathbf{E}_{2}$ :

$$
\mathbf{E}_{2}=\frac{\mathbf{b} \wedge \mathbf{a}}{\sin \theta}
$$

R can be further simplified by substituting (10) into (8):

$$
\mathbf{R}=\cos \theta-\mathbf{E}_{2} \sin \theta \text {. }
$$

The expression is similar to the polar decomposition of a complex number with the unit imaginary replaced by the 2-blade $\mathbf{E}_{2}$. It can also be written as the exponentials of $\mathbf{E}_{2}$ :

$$
\mathbf{R}=e^{-E_{2} \theta}
$$

This formalism is more useful for the log-space of rotors is linear. Up to this point, the angle of rotation in the $\mathbf{b} \wedge \mathbf{a}$ plane remains to be determined. We split $\mathbf{x}$ into part $\left(\mathbf{x}_{p}\right)$ parallel to $\mathbf{b} \wedge \mathbf{a}$-plane and part $\left(\mathbf{x}_{o}\right)$ orthogonal to $\mathbf{b} \wedge \mathbf{a}$-plane. Then, $\mathbf{x}_{o}$ is not affected by application $\mathbf{R}$. And we infer that the

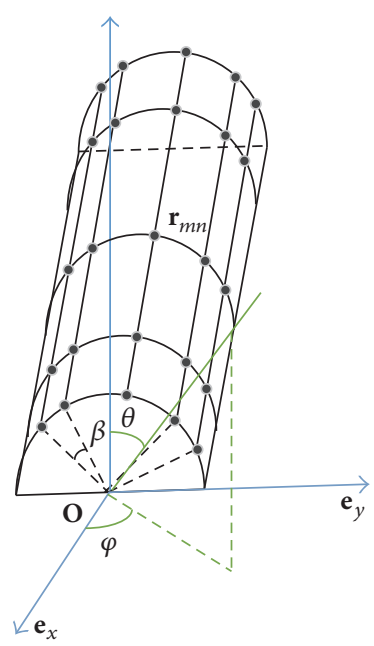

FIGURE 4: The cylindrical conformal array consisting of $M \times N$ short dipoles.

rotation must be in $\mathbf{b} \wedge \mathbf{a}$-plane. As stated above, the rotation consists of two successive reflections which are orthogonal (angle-preserving) transformations. Thus, it allows us to pick any vector in $\mathbf{b} \wedge \mathbf{a}$-plane to determine the angle. Without loss of generality, we choose vector a and construct the "sandwich product" $\mathbf{R a R}^{-1}$ as shown in (7):

$$
\mathbf{R a R}^{-1}=\mathbf{b a a a}^{-1} \mathbf{b}^{-1}=\mathbf{b a b}^{-1}
$$

where $\mathbf{b} \mathbf{a b} \mathbf{b}^{-1}$ is the reflection of $\mathbf{a}$ in $\mathbf{b}$. From this, it is clear that the rotation must be over twice the angle between a and $\mathbf{b}$, since the angle between $\mathbf{a}$ and $\mathbf{b a b} \mathbf{b}^{-1}$ is twice the angle between $\mathbf{a}$ and $\mathbf{b}$. The negative signature in (12) represents the rotation direction.

Consequently, if we want to rotate a vector counterclockwise by a specific angle, we only need to apply the rotor to the vector.

\section{GA-MUSIC Algorithm}

3.1. Array Manifold Modeling Based on GA-MUSIC. In this subsection, we will combine the geometric algebra with MUSIC to estimate the DOA. To illustrate the versatility of this algorithm, we consider $M \times N$ cylindrical conformal array as shown in Figure 4 . The array contains $N$ uniformly spaced rings on the surface. In addition, there are $M$ dipoles distributed on each ring. We assume that each dipole is a short dipole whose output voltage is proportional to the electric field along the dipole. The angle between two consecutive elements on the same ring is $\beta$.

Assume that there are $P$ far-field narrow band polarization sources. $\theta_{p}$ represents $p$ th signal elevation angle which is measured down from $\mathbf{e}_{z}$-axis. $\varphi_{p}$ indicates $p$ th signal azimuth angle and is measured counterclockwise from $\mathbf{e}_{x}$ axis. The polarization ellipse of $p$ th signal is depicted by constants $\gamma_{p}$ and $\eta_{p}$, representing the auxiliary polarization angle and the polarization phase difference [10], respectively. 


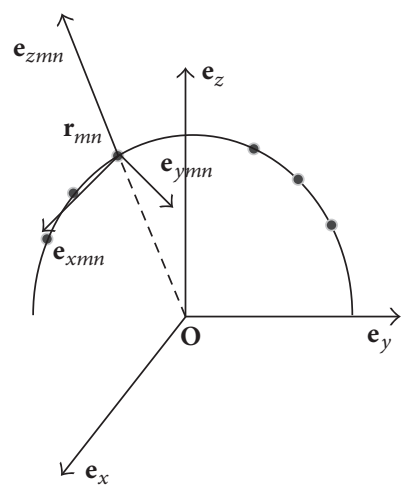

Figure 5: The local coordinate of $(m, n)$ th element.

The array element spatial phase matrix of the $p$ th signal can be described as follows:

$$
\Upsilon_{p}=\Upsilon_{\theta_{p}, \varphi_{p}}=\left[\begin{array}{lll}
u_{p, 1} & & \\
& \ddots & \\
& & u_{p, P}
\end{array}\right],
$$

where $\Upsilon_{p}$ is the diagonal matrix depicting the output signal spatial coherent structures. $k$ th diagonal element, $u_{p, k}=e^{-j 2 \pi\left(\varepsilon_{s}^{T}\left(\theta_{p}, \varphi_{p}\right) \mathbf{r}_{k}\right) / \lambda_{p}}$, represents the space phase factor about $p$ th array element. Among which, $\boldsymbol{\varepsilon}_{s}\left(\theta_{p}, \phi_{p}\right)=$ $-\left[\sin \theta_{p} \cos \varphi_{p}, \sin \theta_{p} \sin \varphi_{p}, \cos \theta_{p}\right]^{\mathrm{T}}$ and $\lambda_{p}$ are $p$ th signal propagation vector and the wavelength, respectively. Symbol $\mathbf{r}_{k}$ is the element location vector and $(\cdot)^{\mathrm{T}}$ denotes the transpose operator.

It is worthwhile to note that the aforementioned element spatial phase matrix, $\Upsilon_{p}$, is derived under the global coordinate system. The azimuth and elevation are defined in Figure 4 as well. However, due to the effects of the curvature of conformal carriers, the local coordinate system is distinct from the global one. As stated above, the rotor can be used to realize the rotation between the two coordinate systems. Thus, we define the local coordinate system of $(m, n)$ th element as shown in Figure 5.

$\mathbf{e}_{x m n}$-axis is the same as $\mathbf{e}_{x}$-axis in the global coordinate system, $\mathbf{e}_{z m n}$ is normal to the element surface, and $\mathbf{e}_{y m n}$ is tangent to the surface so as to form a right-handed coordinate system. Naturally, transforming the global coordinate into the local one is equivalent to rotating the global coordinate around $\mathbf{e}_{x}$-axis. From the last section, we have known that (12) denotes the rotation in $\mathbf{b} \wedge \mathbf{a}$-plane with twice the value of $\theta$. The rotation angle is

$$
\xi=(m-1) \beta-\frac{M-1}{2} \beta=\left(m-\frac{M+1}{2}\right) \beta .
$$

Substituting $\mathbf{e}_{z}$ and $\mathbf{e}_{y}$ for $\mathbf{b}$ and $\mathbf{a}$, respectively, then the rotor is

$$
\mathbf{R}_{m n}=e^{-\left(\mathrm{e}_{z} \wedge \mathrm{e}_{y}\right)(\xi / 2)} .
$$

In addition, $\mathbf{e}_{x}, \mathbf{e}_{y}$, and $\mathbf{e}_{z}$ are orthogonal to each other and the inner products between them are zero. So,

$$
\mathbf{e}_{z} \wedge \mathbf{e}_{y}=\mathbf{e}_{z} \mathbf{e}_{y} .
$$

According to the antisymmetry property of the outer product,

$$
\mathbf{e}_{z} \mathbf{e}_{y}=\mathbf{e}_{z} \wedge \mathbf{e}_{y}=-\mathbf{e}_{y} \wedge \mathbf{e}_{z}=-\mathbf{e}_{y} \mathbf{e}_{z} .
$$

Adopting $\mathbf{E}_{3}=\mathbf{e}_{x} \mathbf{e}_{y} \mathbf{e}_{z}$ as the pseudoscalar in the threedimensional space, (16) can be further simplified:

$$
\mathbf{R}_{m n}=e^{\mathbf{E}_{3} \mathbf{e}_{x}(\xi / 2)}
$$

Through (7), we obtain the standard orthogonal basis in the local coordinate, and the specific calculation process can refer to [11]. Here, the results are given directly:

$$
\begin{aligned}
\mathbf{e}_{x m n}= & \mathbf{R}_{m n} \mathbf{e}_{x} \mathbf{R}_{m n}^{-1}=\mathbf{e}_{x} \\
\mathbf{e}_{y m n}= & \mathbf{R}_{m n} \mathbf{e}_{y} \mathbf{R}_{m n}^{-1} \\
= & \cos \left[\left(m-\frac{M+1}{2}\right) \beta\right] \mathbf{e}_{y} \\
& -\sin \left[\left(m-\frac{M+1}{2}\right) \beta\right] \mathbf{e}_{z} \\
\mathbf{e}_{z m n}= & \mathbf{R}_{m n} \mathbf{e}_{z} \mathbf{R}_{m n}^{-1} \\
= & \sin \left[\left(m-\frac{M+1}{2}\right) \beta\right] \mathbf{e}_{y} \\
& +\cos \left[\left(m-\frac{M+1}{2}\right) \beta\right] \mathbf{e}_{z} .
\end{aligned}
$$

Referring back to (14), the remaining unknown variable is $\mathbf{r}_{k}$. The position vector of $(m, n)$ th element in the global frame is

$$
\begin{aligned}
\mathbf{r}_{m n}= & (-n \delta) \mathbf{e}_{x}+\left(R \sin \left(m-\frac{M+1}{2}\right) \beta\right) \mathbf{e}_{y} \\
& +\left(R \cos \left(m-\frac{M+1}{2}\right) \beta\right) \mathbf{e}_{z} \\
& m=1, \ldots, M ; n=1, \ldots, N,
\end{aligned}
$$

where $\delta$ means the spacing between adjacent rings. Thus, $\Upsilon_{p}$ can be obtained. If $g$ is the gain when the signal perfectly matched the antenna polarization, then the element of generalized polarization sensitive matrix $\mathbf{Q}$ can be represented as

$$
=g\left[\cos \left(\varphi^{\prime}\right) \sin \left(\theta^{\prime}\right) \sin \left(\varphi^{\prime}\right) \sin \left(\theta^{\prime}\right) \cos \left(\theta^{\prime}\right)\right]^{\mathrm{T}},
$$

where $\theta^{\prime}$ and $\varphi^{\prime}$ indicate the elevation and azimuth pointing directions of the short dipole.

According to this, we can get the array manifold:

$$
\mathbf{a}_{p}=\mathbf{a}_{\theta_{p}, \phi_{p}, \gamma_{p}, \eta_{p}}=\Upsilon_{p} \mathbf{Q} \Psi_{p} \mathbf{h}_{p},
$$

where $\mathbf{h}_{p}$ is $p$ th signal polarization vector [12] and can be described by $\gamma_{p}$ and $\eta_{p}$, that is, $\mathbf{h}_{p}=\left[\begin{array}{ll}\cos \gamma_{p} & \sin \gamma_{p} e^{j \eta_{p}}\end{array}\right]^{\mathrm{T}} . \Psi_{p}$ 
is the steering vector of the angle field [13] and is independent of the space location:

$$
\Psi_{p}=\left[\begin{array}{cc}
-\sin \varphi_{p} & \cos \theta_{p} \cos \varphi_{p} \\
\cos \varphi_{p} & \cos \theta_{p} \sin \varphi_{p} \\
0 & -\sin \theta_{p}
\end{array}\right] .
$$

The received signals of the array are a superposition of the response of each signal, and the output can be expressed as

$$
\mathbf{x}(t)=\sum_{p=1}^{P} \mathbf{a}_{p} s_{p}(t)+\mathbf{n}(t),
$$

where $s_{p}(t)$ is $p$ th signal and $\mathbf{n}(t)$ is assumed to be zero mean, complex Gaussian processes statistically independent of each other, with covariance $\sigma_{n}{ }^{2}$.
Up to the present, we have perfectly applied the geometric algebra to the derivation of the steering vector of the conformal array and acquired the GA-MUSIC algorithm. This is also the focus of our work. As for the content of constructing the spatial spectrum and searching the peak, the readers can refer to literature [14]. As the spatial location of the short dipole is arbitrary when introducing the "rotors," the GA-MUSIC is not limited to any specific array geometry.

3.2. Comparing with the Conventional Modeling Using Euler Angle. For better understanding of the superiority of the geometric algebra in modeling the conformal array, we will briefly introduce the conventional methods of analysis based on Euler angle. In general, the transformations from the element local coordinates to the array global coordinates can be realized by three successive Euler rotations [2]. The corresponding rotation matrix can be written as

$$
\begin{aligned}
\mathbf{R}(C, D, F) & =\mathbf{R}_{x}(C) \mathbf{R}_{y}(D) \mathbf{R}_{z}(F)=\left[\begin{array}{ccc}
1 & 0 & 0 \\
0 & \cos C & -\sin C \\
0 & \sin C & \cos C
\end{array}\right]\left[\begin{array}{ccc}
\cos D & 0 & -\sin D \\
0 & 1 & 0 \\
\sin D & 0 & \cos D
\end{array}\right]\left[\begin{array}{ccc}
\cos F & -\sin F & 0 \\
\sin F & \cos F & 0 \\
0 & 0 & 1
\end{array}\right] \\
& =\left[\begin{array}{ccc}
\cos D \cos F & -\cos D \sin F & -\sin D \\
\cos C \sin F-\cos F \sin C \sin D & \cos C \cos F+\sin C \sin D \sin F & -\cos D \sin C \\
\sin C \sin F+\cos C \cos F \sin D & \cos F \sin C-\cos C \sin D \sin F & \cos C \cos D
\end{array}\right],
\end{aligned}
$$

where $C, D, F$ are three successive Euler rotation angles about $\mathbf{e}_{x}$-axis, $\mathbf{e}_{y}$-axis, and $\mathbf{e}_{z}$-axis, respectively. $\mathbf{R}_{x}(C), \mathbf{R}_{y}(D)$, and $\mathbf{R}_{z}(F)$ are the corresponding Euler rotation matrices. Note that, for cylindrical conformal array, two successive Euler rotations are usually sufficient [2]. The third Euler rotation matrix is added here to account for some irregular or complex conformal arrays. Moreover, as shown in (28), the rotation matrix is invertible. Thus, the inversion is taken with respect to $\mathbf{R}(C, D, F)$, resulting in

$$
\begin{aligned}
\mathbf{R}(C, D, F)^{-1} & =\mathbf{R}_{z}^{-1}(F) \mathbf{R}_{y}^{-1}(D) \mathbf{R}_{x}^{-1}(C)=\left[\begin{array}{ccc}
\cos F & \sin F & 0 \\
-\sin F & \cos F & 0 \\
0 & 0 & 1
\end{array}\right]\left[\begin{array}{ccc}
\cos D & 0 & \sin D \\
0 & 1 & 0 \\
-\sin D & 0 & \cos D
\end{array}\right]\left[\begin{array}{ccc}
1 & 0 & 0 \\
0 & \cos C & \sin C \\
0 & -\sin C & \cos C
\end{array}\right] \\
& =\left[\begin{array}{ccc}
\cos D \cos F & \cos C \sin F-\cos F \sin C \sin D & \sin C \sin F+\cos C \cos F \sin D \\
-\cos D \sin F & \cos C \cos F+\sin C \sin D \sin F & \cos F \sin C-\cos C \sin D \sin F \\
-\sin D & -\cos D \sin C & \cos C \cos D
\end{array}\right] .
\end{aligned}
$$

From (28) and (29), it is not hard to find that

$$
\mathbf{R}(C, D, F)^{-1}=\mathbf{R}(C, D, F)^{T} .
$$

So, $\mathbf{R}(C, D, F)$ is the orthogonal matrix. Then, taking the transformation from the element local coordinates to the array global coordinates is equivalent to taking the transposition/inversion with respect to the aforementioned rotation matrix. If we model the conformal array adopting the Euler angle, three matrix multiplications and one matrix transposition are involved for each element.
In practical applications, the matrix operations are essentially the multiplication and addition operations between elements. To quantify what we mean by this, the amounts of multiplication and addition operations of the two algorithms (i.e., GA-MUSIC and Euler angle) are computed, respectively, as shown in Table 2. Suppose that one matrix transposition is regarded as one multiplication or addition operation. As is known to all, the multiplication between two $3 \times 3$ matrices requires $9 \times 3$ multiplications and $9 \times 2$ additions. For convenience, the multiplication operation and the addition operation are collectively referred to as the operation. Then, 
TABLE 2: The computational complexity of GA-MUSIC and Euler angle.

\begin{tabular}{lcccc}
\hline & Multiplications & Additions & Transpositions & Operations \\
\hline Euler angle & $2 \times 9 \times 3 \times M N$ & $2 \times 9 \times 2 \times M N$ & $M N$ & $91 \times M N$ \\
GA-MUSIC & $4 \times M N$ & $2 \times M N$ & 0 & $6 \times M N$ \\
\hline
\end{tabular}

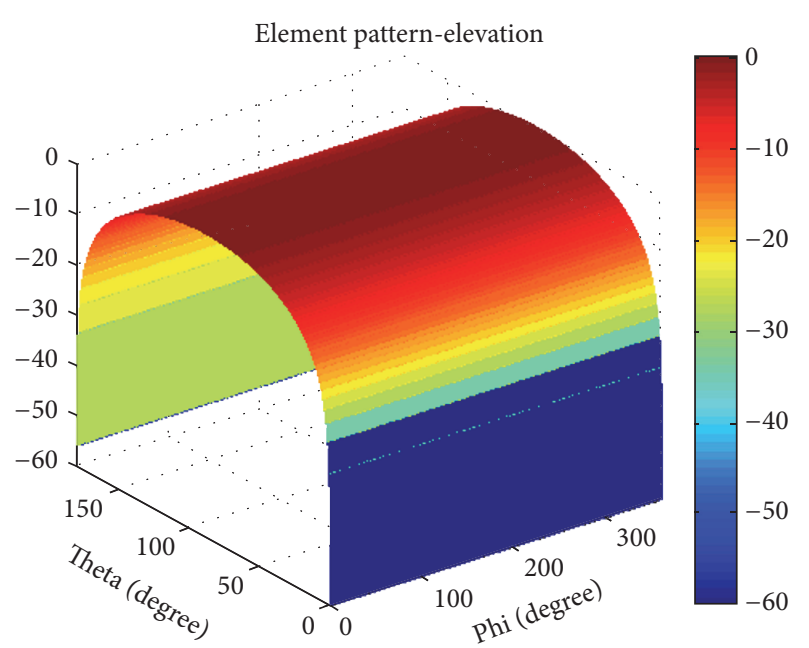

(a)

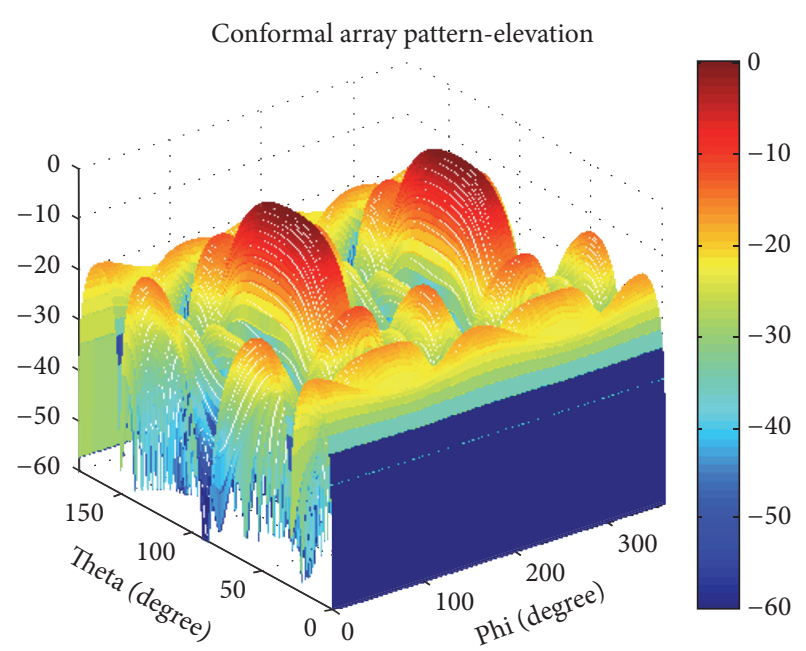

(b)

FIgURE 6: Pattern analysis: (a) pattern of single short dipole and (b) pattern of cylindrical conformal array.

the calculation of $(28)$ contains $2 \times 9 \times 3+2 \times 9 \times 2$ operations. For the conformal array consisting of $M \times N$ elements, the transformation between different coordinates involves $91 \times$ $M N$ operations. In contrast to the Euler angle, GA-MUSIC avoids the cumbersome matrix transformations.

From (20)-(22), we know that $\mathbf{e}_{y m n}$ and $\mathbf{e}_{z m n}$ are independent of $\mathbf{e}_{x}$. Moreover, $\mathbf{e}_{x m n}$ can be obtained directly from (20) without extra computations. Thus, (21)-(22) can be rewritten in $2 \times 2$ matrix. While applying the rotor to construct the array manifold, the computational process is equivalent to 2 $\times 2$ matrix multiplied by $2 \times 1$ vector. Then, the operations for each element involve 4 multiplications and 2 additions. The total amount of operations is $6 \times M N$. Thus, the latter method largely decreases the computational complexity.

To sum up, the Euler rotation and its matrix representation cannot intuitively display the whole procedure. Moreover, as the configuration of the conformal array becomes more irregular and complex, the level of complexity involved in the transformations and the number of calculations required increase significantly.

\section{Simulation Results}

In this section, Monte-Carlo simulation experiments are employed to verify the effectiveness of the GA-MUSIC algorithm. The array structure is shown in Figure 4. We select $M$ and $N$ as 4 and 2, respectively, for the validations. The pattern of single short dipole is shown in Figure 6(a), while the cylindrical conformal array pattern is displayed in Figure 6(b). Compared with the existing methods, adopting the geometric algebra will be more simple and intuitive. For the solved array pattern or manifold is the superposition of various elements, the proposed method can be applied to arbitrary array structures. This also proves the superiority of the rotor in solving conformal problems.

Next, the performance of the GA-MUSIC algorithm is to be verified. We make some notations. Firstly, the absolute value of the differences between the estimated mean and the true value is regarded as the deviation. Secondly, the RMSE is utilized as the performance measure. Under these premises, 100 independent simulation experiments are carried out. The RMSE is defined as

$$
\operatorname{RMSE}=\sqrt{\frac{1}{100} \sum_{i=1}^{100}\left[\left(\widehat{\theta}_{i}-\theta_{i}\right)^{2}+\left(\widehat{\varphi}_{i}-\varphi_{i}\right)^{2}\right]}
$$

where $\left\{\widehat{\theta}_{i}, \widehat{\varphi}_{i}\right\}$ are the estimates of elevation angles and azimuth angles, respectively, at $i$ th run. Wang's method [2] and Qun's method [4] are included for comparison.

Provided that there are three signals that can be received, the incident angles are $\left(15^{\circ}, 40^{\circ}\right),\left(35^{\circ}, 10^{\circ}\right)$, and $\left(60^{\circ}, 65^{\circ}\right)$, respectively. The corresponding polarization auxiliary angle and the polarization phase difference are $\left(20^{\circ}, 25^{\circ}\right),\left(50^{\circ}\right.$, $\left.45^{\circ}\right)$, and $\left(65^{\circ}, 65^{\circ}\right)$. The snapshot, $K$, is selected as 100 . Figure 7 (a) shows the simulation results of the GA-MUSIC algorithm. The position of the spectrum peak represents the corresponding signal DOA. Intuitively, the estimation accuracy of the GA-MUSIC algorithm is high.

We assume that there are nine distinct signals impinging on the cylindrical conformal array at the same time. The 


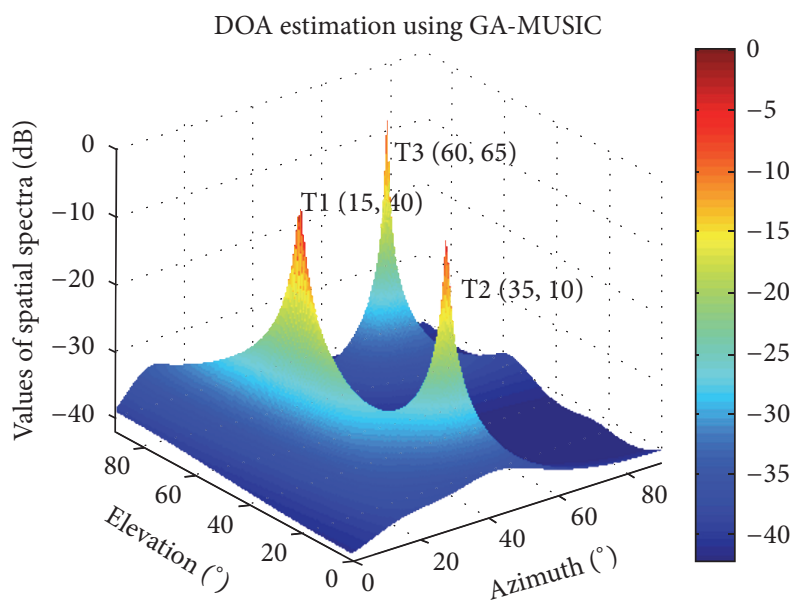

(a)

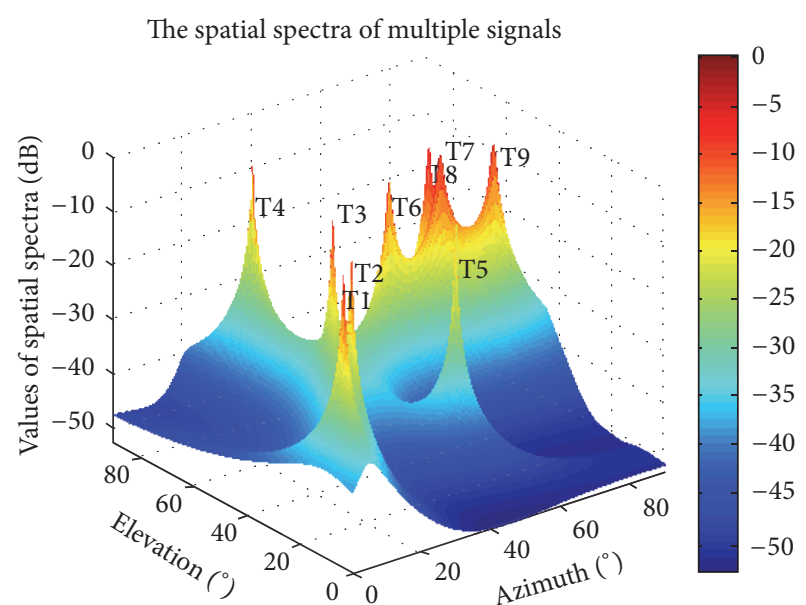

(b)

FIGURE 7: The performance of GA-MUSIC algorithm: (a) DOA estimation using GA-MUSIC and (b) the spatial spectra of multiple signals.

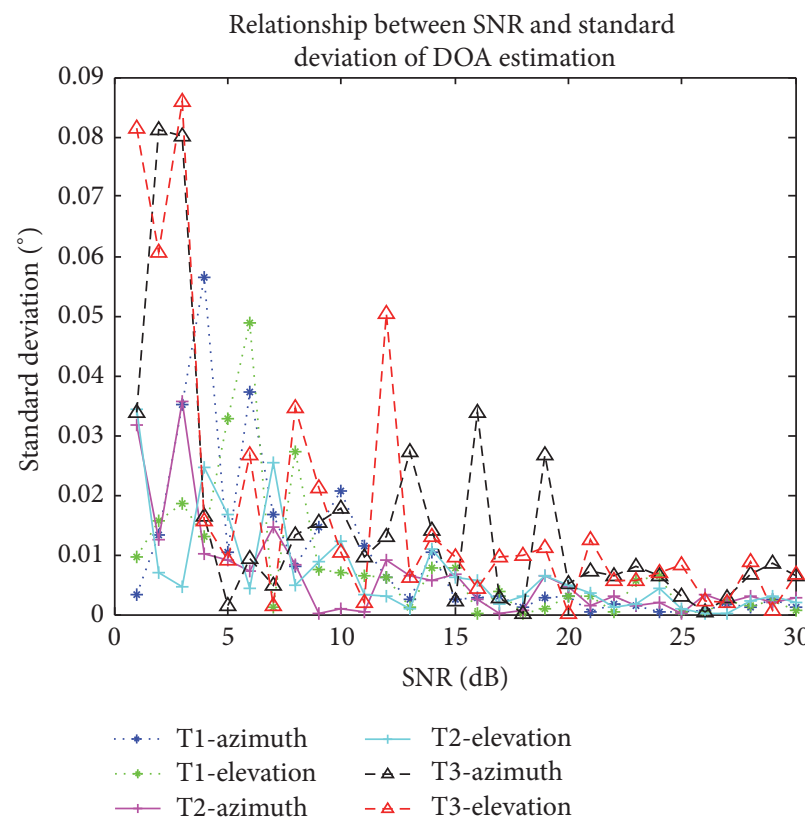

(a)

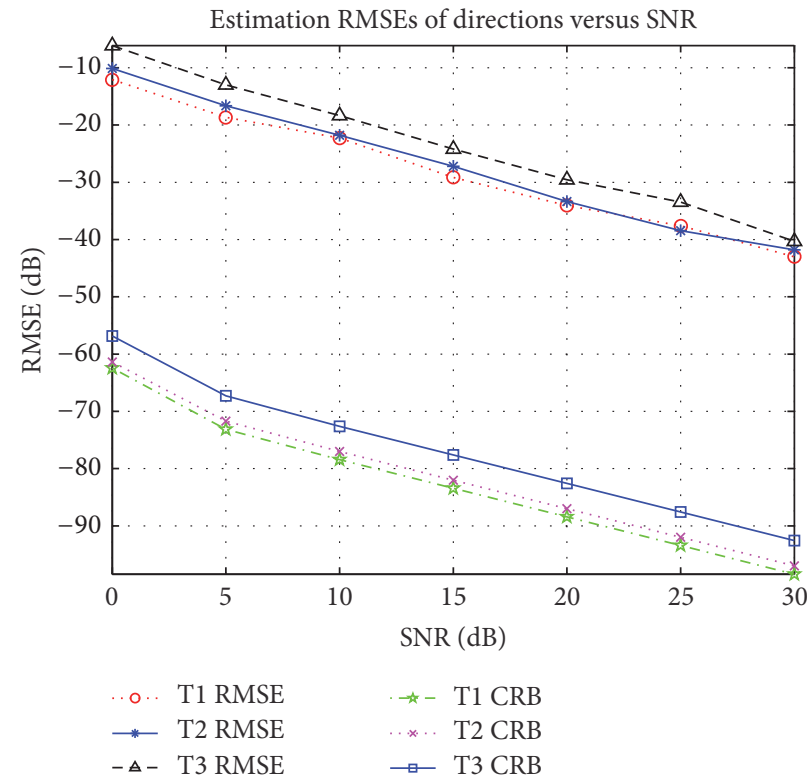

(b)

FIgURE 8: The DOA estimation accuracy with $K=100$ : (a) deviation of estimates versus SNR and (b) the RMSE and CRB versus SNR.

spatial spectra are shown in Figure 7(b). It is obvious that the method still works effectively when the number of incident signals is larger than that of the array elements.

Figure 8 displays the performance with a varying SNR from $0 \mathrm{~dB}$ to $30 \mathrm{~dB}$. Among them, Figure 8 (a) displays the relationship between SNR and deviation of DOA estimation, while Figure 8(b) reveals the Cramer-Rao Lower Bound (CRLB) and the RMSE versus the SNR, respectively. It is clear that the deviation varies inversely with SNR. The higher the SNR, the lower the deviation. The trend of the variance of the CRLB with SNRs is the same as the RMSE, which is expected. Obviously, in the engineering design, the higher the SNR, the better the estimation performance that we can obtain.
Note that, since statistical data have some randomness, the simulation curves in Figure 8(a) are not smooth and do not decline monotonically.

To proceed further, we increase the number of snapshots to 200 and leave the other conditions unchanged. The respective results are shown in Figure 9. Compared with Figure 8 , both the deviation and $\mathrm{CRB}$ were improved. If we choose the point at some SNR, we can find that the CRB of Figure 8 is nearly twice as much as of Figure 9. For example, the CRB of Target 1 is $-82.58 \mathrm{~dB}$ when the SNR is $20 \mathrm{~dB}$ as shown in Figure 8(b), while, in Figure 9(b), the value is $-86.08 \mathrm{~dB}$. In fact, these improvements can be predicted from the derivation of CRLB. For details on the specific 


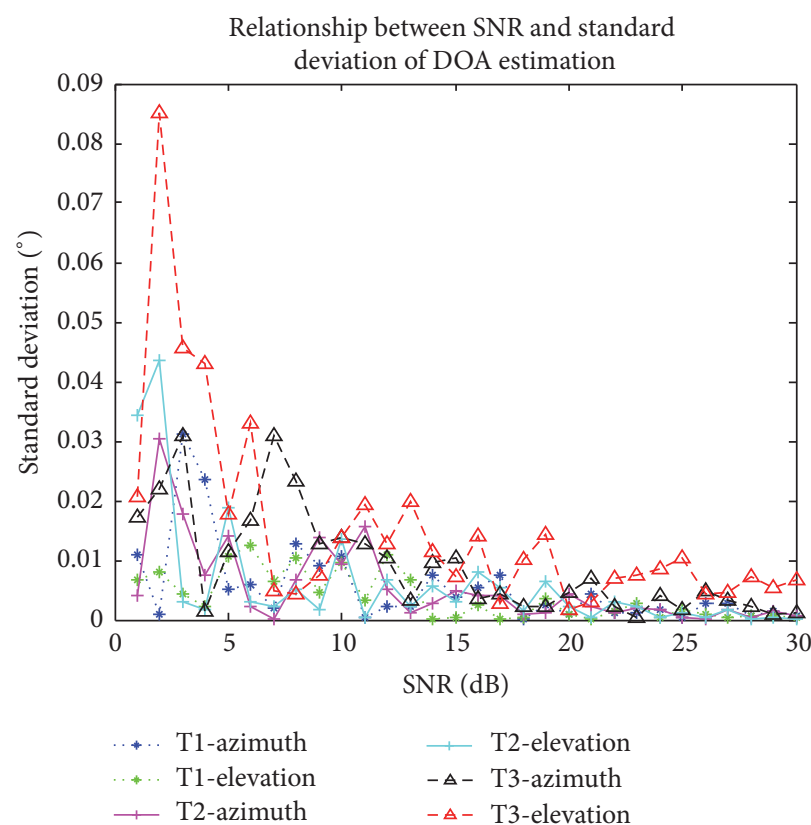

(a)

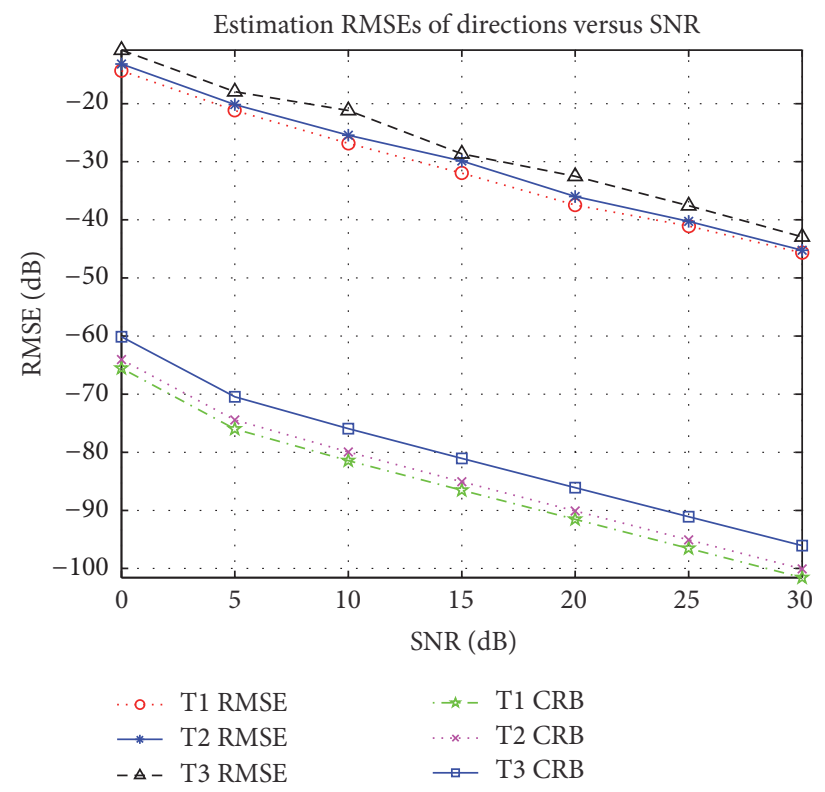

(b)

FIGURE 9: The DOA estimation accuracy with $K=200$ : (a) deviation of estimates versus SNR and (b) the RMSE and CRB versus SNR.

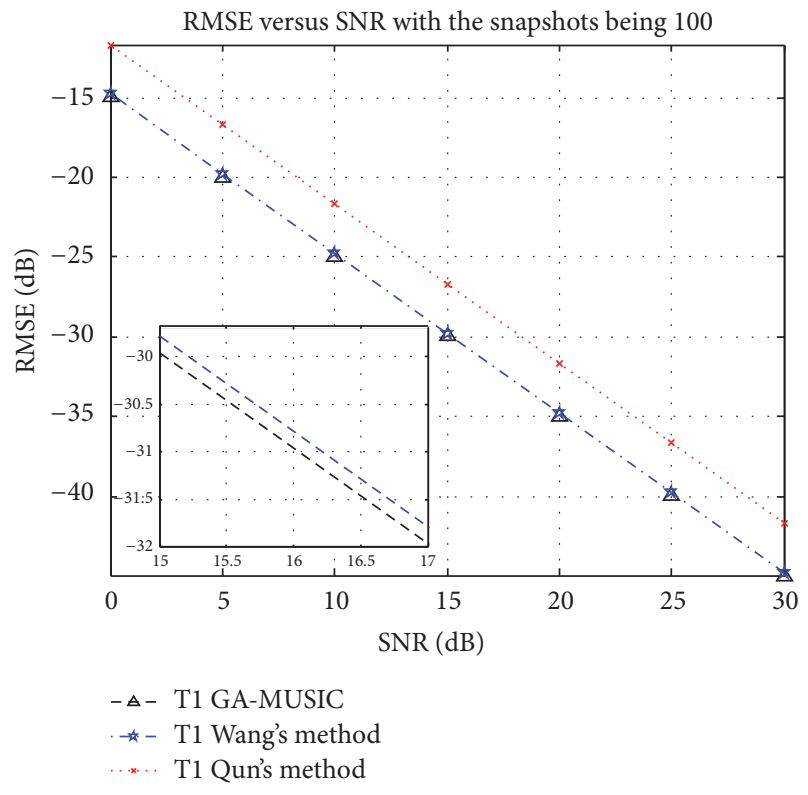

(a)

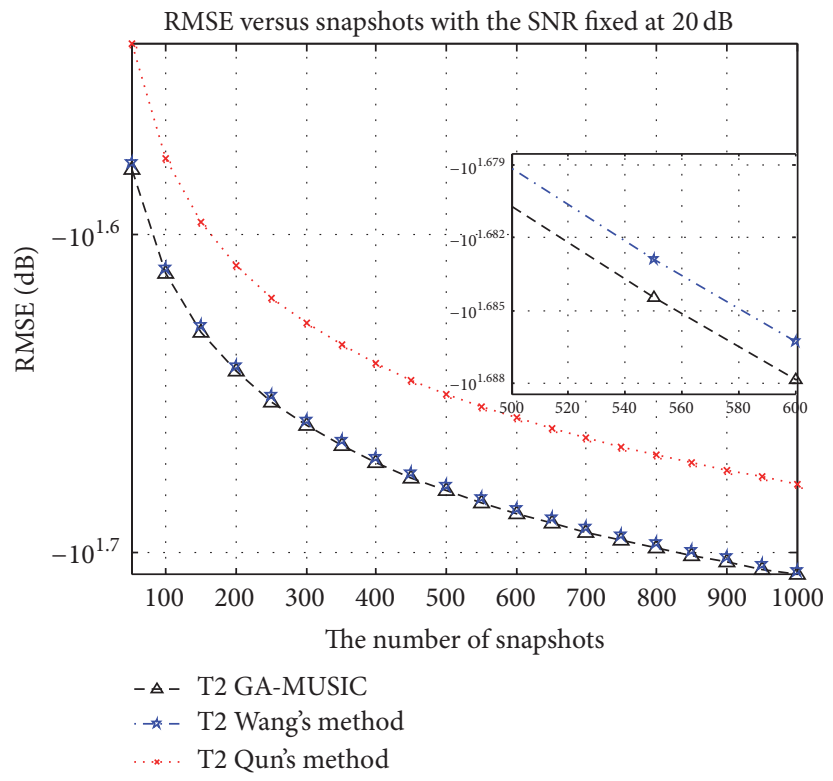

(b)

FIGURE 10: Improvements of GA-MUSIC: (a) RMSE versus SNR with the snapshots being 100 and (b) RMSE versus the snapshots with the SNR fixed at $20 \mathrm{~dB}$.

derivation process, see the work of Stoica and Nehorai $[15,16]$. The number of snapshots can be extracted from the Fisher information matrix. Moreover, the CRLB is found as the element of the inverse of that matrix. So, we can conclude that CRLB is inversely proportional to $K$. Thus, the estimation precision will be higher.

Figure 10 shows the improvements of GA-MUSIC over existing algorithms, such as Qun's method [4] and Wang's method [2]. We study the performance with a varying SNR from $0 \mathrm{~dB}$ to $30 \mathrm{~dB}$ and the performance with the number of snapshots varying from 100 to 1000 , respectively. Without loss of generality, we select the first source (T1) and the second source (T2), respectively, to verify it. As shown in Figure 10(a), the proposed method outperforms Qun's method by exploiting the polarization information of the received data. Moreover, the elliptic covariance matrix of 
the GA-MUSIC is nonzero which increases the information utilization rates as well.

The RMSE of the GA-MUSIC is close to that of the Wang's method. The reason is that the estimation accuracy mainly depends on the steering vector. Both the Euler rotation angle and the geometric algebra can be used to derive the steering vector. As previously mentioned, the array manifold was obtained by using the rotor in this paper. However, in [2], Wang derived the array manifold by means of the Euler rotation angle. Therefore, in this case, both methods exhibit the same performance. From Section 3.2, we clearly know the GA-MUSIC is superior to the Euler rotation angle in computational complexity.

Figure 10(b) illustrates the RMSE versus the number of snapshots with the SNR fixed at $20 \mathrm{~dB}$. Compared with Figure 10(a), we can draw similar conclusions.

\section{Conclusion}

In this paper, we proposed a novel algorithm named GAMUSIC to estimate the DOA for cylindrical conformal array, which combines the geometric algebra with MUSIC. Compared with existing methods based on Euler rotation angle, it avoids complex matrix transformations and largely decreases the computational complexity. In contrast to the method introduced in the literature [8], our presented method has a strong commonality which can be used for arbitrary array structure. In addition, it can also be suited for the case that the number of polarized signals is larger than that of the array elements. At last, the simulated results verify the effectiveness of the GA-MUSIC algorithm.

\section{Appendix}

Here, we derive the calculation process of (4).

From (1)-(3), we get

$$
\begin{aligned}
\mathbf{x y x} & =(\mathbf{x} \bullet \mathbf{y}+\mathbf{x} \wedge \mathbf{y}) \mathbf{x}=(\mathbf{x} \bullet \mathbf{y}) \mathbf{x}+(\mathbf{x} \wedge \mathbf{y}) \mathbf{x} \\
& =(\mathbf{x} \bullet \mathbf{y}) \mathbf{x}+\frac{\mathbf{x y}-\mathbf{y x}}{2} \mathbf{x}=(\mathbf{x} \bullet \mathbf{y}) \mathbf{x}+\frac{\mathbf{x y}}{2} \mathbf{x}-\frac{\mathbf{y x}}{2} \mathbf{x} \\
& =(\mathbf{x} \bullet \mathbf{y}) \mathbf{x}+\frac{\mathbf{x y}}{2} \mathbf{x}-\frac{\mathbf{y}}{2} .
\end{aligned}
$$

Thus,

$$
\frac{\mathbf{x y x}}{2}=(\mathbf{x} \cdot \mathbf{y}) \mathbf{x}-\frac{\mathbf{y}}{2}
$$

Up to the present, (4) has been obtained.

\section{Competing Interests}

The authors declare that they have no competing interests.

\section{Acknowledgments}

This project was supported by the National Natural Science Foundation of China (Grant no. 61302017).

\section{References}

[1] L. Josefsson and P. Persson, Conformal Array Antenna Theory and Design, IEEE Press Series on Electromagnetic Wave Theory, 2006.

[2] B.-H. Wang, Y. Guo, Y.-L. Wang, and Y.-Z. Lin, "Frequencyinvariant pattern synthesis of conformal array antenna with low cross-polarisation," IET Microwaves, Antennas and Propagation, vol. 2, no. 5, pp. 442-450, 2008.

[3] T. Milligan, "More applications of euler rotation angles," IEEE Antennas and Propagation Magazine, vol. 41, no. 4, pp. 78-83, 1999.

[4] Q. Yang, X.-Y. Cao, J. Gao, and W.-Q. Li, "An algorithm of conformal array DOA estimation with unknown source number," in Proceedings of the 3rd Asia-Pacific Conference on Antennas and Propagation (APCAP '14), pp. 502-505, Harbin, China, July 2014.

[5] H. A. Burger, "Use of euler-rotation angles for generating antenna patterns," IEEE Antennas and Propagation Magazine, vol. 37, no. 2, pp. 56-63, 1995.

[6] V. de Sabbata and B. K. Datta, Geometric Algebra and Applications to Physics, CRC Press, Boca Raton, Fla, USA, 2007.

[7] L. Zou, J. Lasenby, and Z. He, "Pattern analysis of conformal array based on geometric algebra," IET Microwaves, Antennas \& Propagation, vol. 5, no. 10, pp. 1210-1218, 2011.

[8] L. Zou, J. Lasenby, and Z. He, "Direction and polarisation estimation using polarised cylindrical conformal arrays," IET Signal Processing, vol. 6, no. 5, pp. 395-403, 2012.

[9] L. Dorst, D. Fontijne, and S. Mann, Geometric Algebra for Computer Science, Morgan Kaufmann, Boston, Mass, USA, 2007.

[10] J. Li and R. T. Compton Jr., "Angle estimation using a polarization sensitive array," IEEE Transactions on Antennas and Propagation, vol. 39, no. 10, pp. 1539-1543, 1991.

[11] D. Hildenbrand, Foundations of Geometric Algebra Computing, Springer, Berlin, Germany, 2013.

[12] G. A. Deschamps, “Techniques for handling elliptically polarized waves with special reference to antennas: part IIgeometrical representation of the polarization of a plane electromagnetic wave," Proceedings of the IRE, vol. 39, no. 5, pp. 540$544,1951$.

[13] A. Nehorai and E. Paldi, "Vector-sensor array processing for electromagnetic source localization," IEEE Transactions on Signal Processing, vol. 42, no. 2, pp. 376-398, 1994.

[14] R. Schmidt, "Multiple emitter location and signal parameter estimation," IEEE Transactions on Antennas and Propagation, vol. 34, no. 3, pp. 276-280, 1986.

[15] P. Stoica and A. Nehorai, "MUSIC, maximum likelihood, and cramer-rao bound," IEEE Transactions on Acoustics, Speech, and Signal Processing, vol. 37, no. 5, pp. 720-741, 1989.

[16] P. Stoica and A. Nehorai, "MUSIC, maximum likelihood, and Cramer-Rao bound: further results and comparisons," IEEE Transactions on Acoustics, Speech, and Signal Processing, vol. 38, no. 12 , pp. $2140-2150,1990$. 


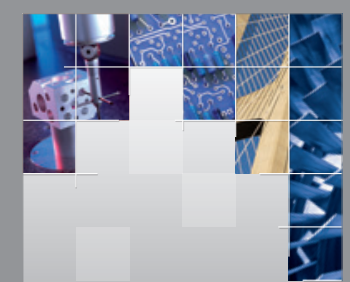

\section{Enfincering}
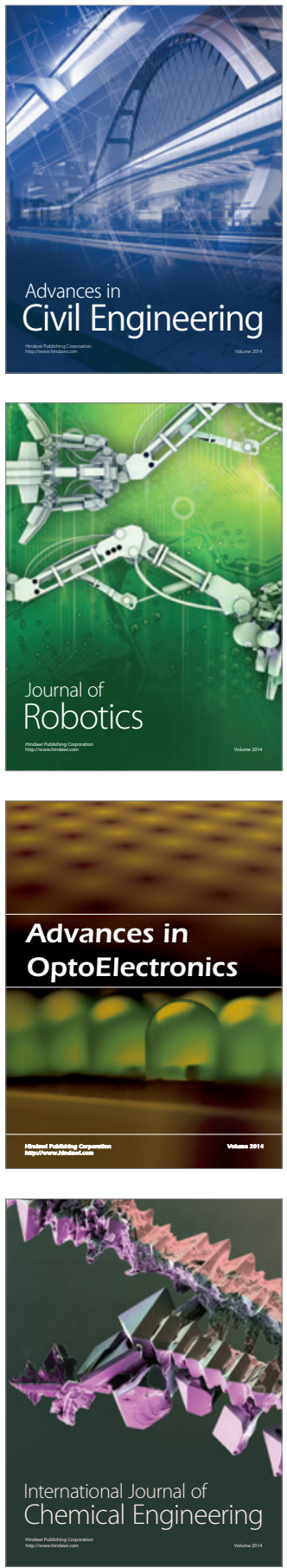

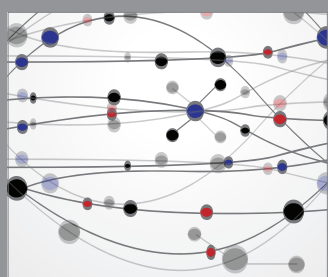

The Scientific World Journal

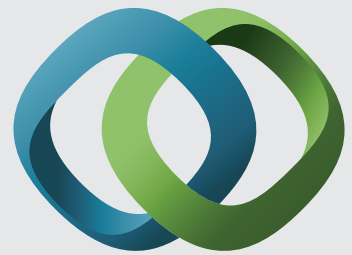

\section{Hindawi}

Submit your manuscripts at

http://www.hindawi.com
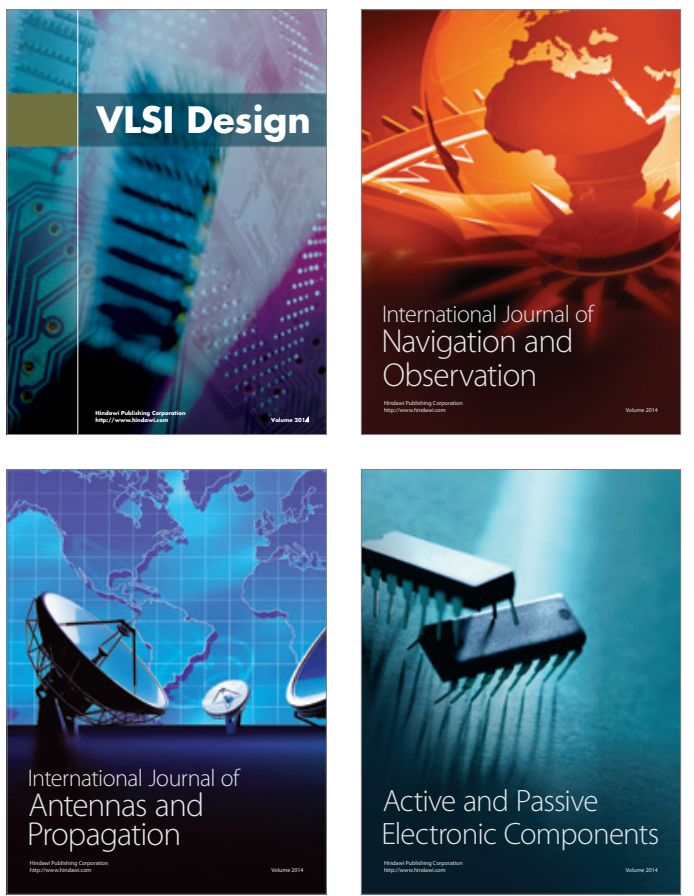
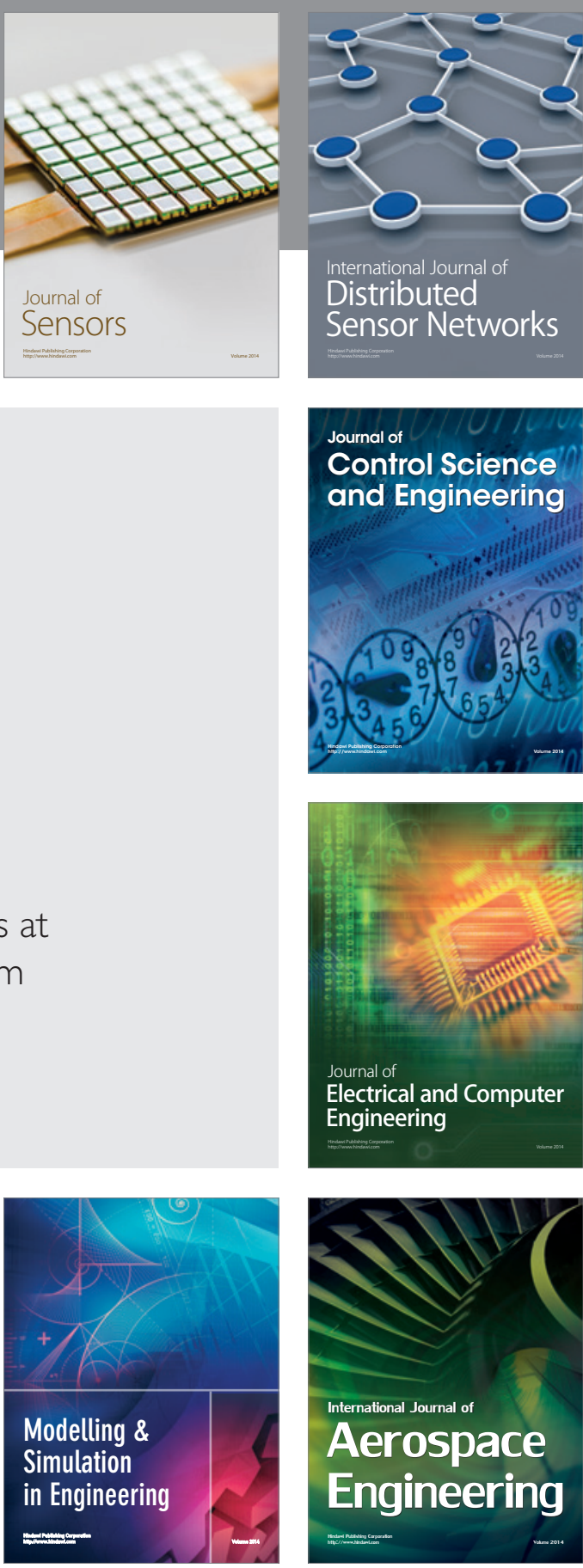

International Journal of

Distributed

Sensor Networks

Journal of

Control Science

and Engineering
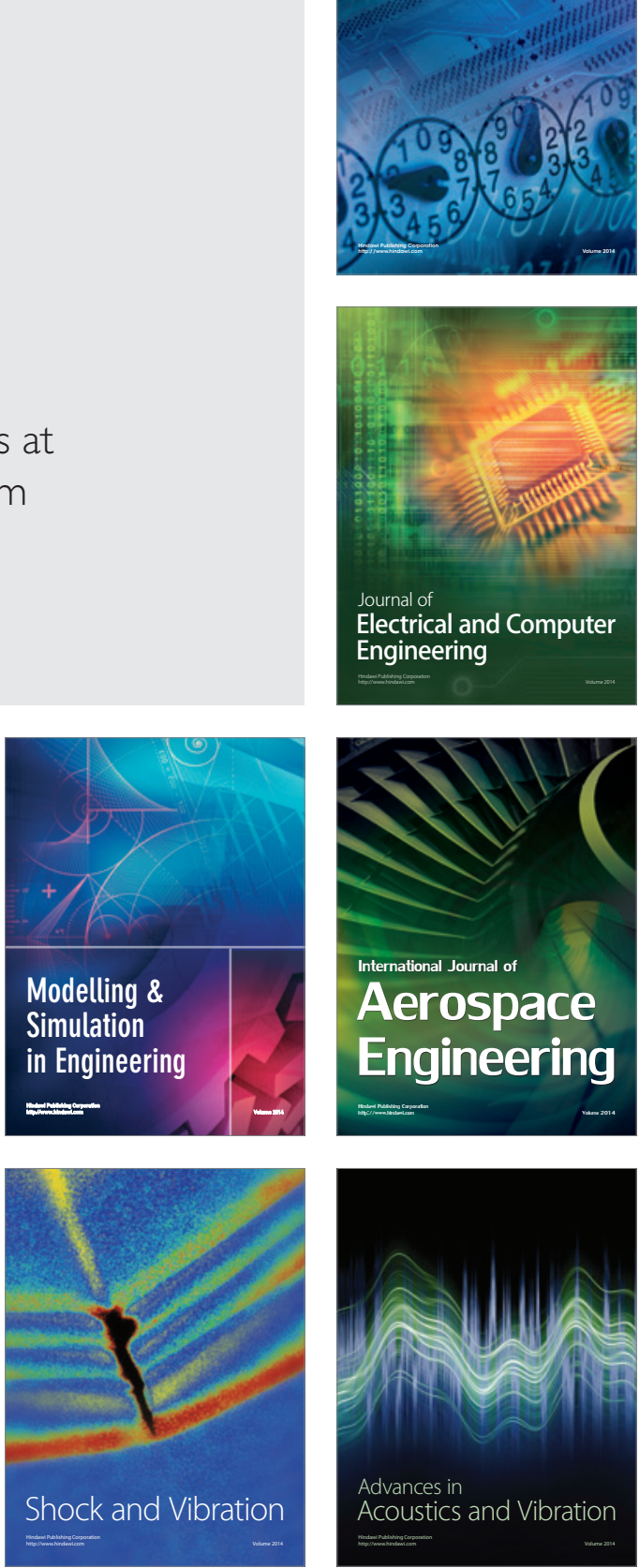